\title{
Dialecto judeo piemontés
}

The dilect judeopiomontes

\author{
Dr. Adolfo Kuznitzky \\ itok@arnet.com.ar \\ Centro de Investigación y Difusión \\ de la Cultura Sefaradi (CIDiCSef) \\ Argentina
}

\section{Resumen}

El largo exilio del pueblo judío, y su consiguiente dispersión geográfica, tuvo decisiva influencia tanto en las lenguas que hablaron, como en otro aspecto lingüístico territorial que es el onomástico.

La particularidad que ofrece el dialecto que estamos tratando es que responde, como los otros, a la fragmentación lingüística existente en Italia. Ese desarrollo de diversos lenguajes dialectales se explica porque esa dispersión idiomática se produjo debido a que el proceso de unificación nacional, con la adopción de idioma único, fue tardío. Así es como existen dialectos judeo-ferrarese, florentino, mantovano, modenese, livornese, etc. Consiguientemente, los hebreos radicados en el Piemonte hablaron el dialecto correspondiente a la región.

No obstante, la dificultad para encontrar la expresión idiomática adecuada y otras necesidades, como sostiene Diena, hizo que se introdujeran algunas palabras hebreas, que se conjugarían con la gramática italiana, un verdadero cuerpo extraño desde el punto de vista lingüístico, salvo que se sostenga la idea de que los idiomas semitas e indoeuropeo tengan un tronco común

En síntesis, se trata de una estructura menor, de exiguo patrimonio léxico, fundamentalmente oral -pese a la existencia de textos literarios-, sin un sistema fonético, 
morfológico o sintáctico propio, con pocos lexemas, que se caracteriza por contar con locuciones y expresiones hebreas insertas en el tejido dialectal piamontés. Consecuentemente, el trato fonético y sintáctico era preferentemente piamontés.

Palabras claves: dialectos judíos, Piemontese, Ashkenaz, Sefarad, Judezmo, yiddish, judeoprovenzal.

\begin{abstract}
The long exile of the Jewish people, and its consequent geographical dispersion, had decisive influence in languages who they spoke, and another territorial linguistic aspect, which is the onomastic.
\end{abstract}

The dialect we are dealing about has the particularity, responding, as others, that is the consecuence of linguistic fragmentation in Italy. The development of several dialectal languages ocurred because it was late when national unificaticon process adopted a single language. This is how there are dialects judeo-ferrarese, florentino, mantovano, modenese, livornese, etc. Accordingly, Jews living in the Piedmont spoke the local dialect.

However, the difficulty to find the proper idiomatic expression and other needs, as Diena holds, made that some hebrew words were introduced, all these changed conformed a strange body from the linguistically point of view, unless,you hold the idea that the Semitic and Indo-European languages have a common trunk.

In synthesis, it is a minor structure of meager heritage lexicon, fundamentally oral - in spite of the existence of literary texts-, without its own phonetic, morphological or syntactic system, with few lexemes, characterized by having voice-overs and Hebrew expressions embedded in the Piedmontese dialect. As a result, phonetic and syntactic treatment was preferably Piedmontese. 
Keywords: Jewish dialects, Piemontese, Ashkenaz, Sepharad, Judezmo, Yiddish, judeoprovenzal. 
En general, los judíos de Italia no hablaban el idioma hebreo, porque no era corriente entre ellos, a excepción de las necesidades del culto,

Questa raccolta de voci ed espressioni della parlata giudeo-piemontese vorrebe fissare le ultime tracce della tradizione linguistica orale, de uso prevalentemente familiare e domestico, tipica degli ebrei piemontese almeno fino aglui inizi di questo secolo. Si tratta de un esiguo patrimonio lessicale; pochi lessemi, locuzioni, modi di dire di origine ebraica inseriti nel tessuto dialettale piemontese senza un proprio sistema fonetico, morfologico e sintattico... Questa parlata (...) a differenza di altre parlate giudaica (...) fu semplicemente un linguaggio orale, mezzo eficace per expressioni cariche di ironia e di allusività, mai puramene descrittivo, spesso tendente all ingiuria e all autocommiserazione, usato forse solo come gergo, per necessità e per abitudine, ma forse anche como mezzo inconsapevole per conservare e difendere una certa alterità culturale, como risposta alle forze disgregatricie del mondo esterno (...) l'uso di un linguaggio particolare era una necessità constante, quotidiana, una risposta al clima de opressione e clausura instaurato (...) Molte sono infatti le vocci e locuzioni che, per il loro esere adoperate in specifiche situazione di sospetto, di paura, di necesità di fuga (zitto non parlare, ecc.)

Diena Paola, Ebrei a Torino

Gli israeliti poi un idioma especiale, de cui sono piutosto - ed a ragione-gelosi. Nel commercio é utilissimo poter comunicare ordini e pensieri senza che $i$ profani capiscano: tutti e gerghi sono nati allo scopo. Oltre a ciò, il linguaggio convenzionale serve como tessera di riconoscimento cuando si è fuori a domicilio...

Alberto Virgilio, Como si parla a Torino 


\section{Introducción}

El largo exilio del pueblo judío, y su consiguiente dispersión geográfica, tuvo decisiva influencia en las lenguas que hablaron, y en otro aspecto lingüístico territorial como el onomástico.

Para dimensionar esas influencias, en primer lugar, repasaremos algunos elementos onomásticos hebreo italianos, porque en los mismos se advierte rápidamente, y en plenitud, las adaptaciones geográfico lingüísticas. En ese terreno, los rasgos principales para determinar las connotaciones que lleven al origen hebreo de un apellido italiano son la toponimia y la movilidad linguística. Esas pautas constituyen pistas solamente; porque, y esto hay que destacarlo, siempre serán aproximaciones, pero casi nunca exteriorizan certezas. Es por ello que son escasos los apellidos italianos -y de otras naciones- incuestionablemente hebreos (podría serlo Sacerdote, traducción de Cohen).

En el área del parentesco lingüístico, lo más común es el “disfraz” idiomático del aspecto antroponímico (significado) en el apellido, y que los lingüistas italianos denominan "travestimento lingüístico". Este proceso consiste en la traducción al italiano de un apelativo hebreo que mantiene su acepción, por ejemplo, Hazan (cantor) Canterini.

Con respecto a la dispersión geográfica, es importante sin duda la toponimia, dado que la proporción de esos apellidos son más frecuentes entre hebreo italianos que entre los gentiles de esta nacionalidad; los primeros registran un porcentaje de un sesenta por ciento, mientras que en el segundo caso llegan a un treinta y siete por ciento. Para Colorni (1989), que es quien estudia este tipo de apellidos, la razón de ese predominio se debe a que esa referencia a la geografía denota la escasez de otras matrices, por la exigüidad de los núcleos humanos que conforman y la uniformidad de su estructura social $^{1}$.

1 Colorni Vittore. "Cognomi Ebraici Italiani a Base Toponomastica Straniera" en Italia Judaica. Roma: 1989. 
En cuanto a los toponímicos que llevaban debemos distinguir, entre los que se relacionan con la geografía de Italia, como, por ejemplo Módena, y los étnicos o gentilicios en los cuales se advierte la influencia lingüística, porque cuando éstos son de origen extranjero, generalmente, han sufrido un proceso de italianización, como Aragona, Algranati (Granada) Foa (Foix), Bedarida (Bedárides), Momigliano (Montmelian), Cavaglione (Cavaglion), Carcassona (Carcassone), Luzzatti (Lausitz), Morpurgo (Marburg), Otolenghi (Ettlingen), Diena (Jena), Treves (Triers), Minzi (Mainz), Grego (griego), Polacco (polaco); Tedeschi (alemán); Gállico (francés).

En relación con el otro caso, se trata de los apellidos con connotaciones lingüísticas hebreas traducidos al italiano, como es el caso de: Bolaffio (Abulaffia), Mestre (maestro o rabbi), Pacifici (Salomón), Sacerdote (Cohen), Buonaventura (Mazl Tov), Leone (Judah), Marco (Mordecai), Guglielmo (Benjamín) Césare (Hezekiah), Macri (Machir), Ascarelli (As Cari), Tranquillo (Manoach), Vivante (Hayyim), Benedicto (Baruch), Grassini (Gerschon), Meatti (Mea), Consiglio (Jecutiel), Consolo (Nachman), Del Vecchio (Zaken), Forti (Hazzak), Angeli (Malechai), Ferro (Barzilai y el oficio correspondiente es uno de los apellidos más "travestidos" del mundo), Pozzo (Beer), Cammeo (Zafir), Colombo (Joná; por ese apellido De Madariaga deduce que Colón podría tener origen judío).

\section{Dialectos judeo italianos}

Para introducirnos en el dialecto que hablaban los hebreos en la zona de Piamonte hay que tener presente, previamente, que los judíos en Italia, como en otras geografías, desarrollaron lenguajes que eran propios de esas naciones, dada la influencia de los idiomas de los países hacia los que migraban que era lo que prevalecía. Así fue como en Ashkenaz (Alemania) hablaron el yiddish, que es, fundamentalmente, el alemán medieval, y en Sefarad (España) el judezmo (más conocido como ladino, pero con la particularidad de que ese lenguaje se formó en lo que sería su segundo exilio, por la expulsión de la península, a partir de la evolución de la lengua española y por el contacto con otras culturas y lenguas). En otras palabras, no es lo mismo el judezmo que hablan los judíos en el norte de África que en Turquía. 
Lo notable, dado que resulta excepcional, es que en Italia recalaron, con frecuencia estimulados oficialmente, numerosos sefarditas expulsados que no hablaron judezmo; ello demostraría el grado de adaptación que tuvieron, que desembocó en el proceso de integración más pleno que vivieron los hebreos en la diáspora.

La particularidad que ofrece el dialecto que estamos tratando es que responde, como los otros, a la fragmentación linguística existente en Italia. El desarrollo de diversos lenguajes dialectales se explica porque esa dispersión idiomática se produjo debido a que el proceso de unificación nacional fue tardío. Así es como existen dialectos judeoferrarese, florentino, mantovano, modenese, livornese (llamado Bagitto y muy influenciado -dado que Livorno tuvo un importante núcleo de población sefardí- por los préstamos linguísticos de la península ibérica), etc. No obstante, existe una controversia, dado que algunos investigadores sostienen que previamente a esa formación regional, así como en Italia hubo un idioma italiano, que luego fue el oficial, también hubo un judeo italiano clásico ${ }^{2}$. Lo concreto es que previamente existió un judeolatín, que podría haber sido el predecesor de todos estos dialectos, pero hay que distinguir entre el judeolatín occidental del meridional; sería éste el que influyó en la formación de esos dialectos, también llamados giudaesco o ghettaiolo término acuñado a partir de la Edad Media en la que la población fue obligada a vivir en los ghetos ${ }^{3}$.

A los efectos del dialecto judeopiemontés, esa distinción del judeolatín tiene importancia, porque el occidental, que no influyó en el judeo italiano, sí incidió en la formación de las lenguas que hablaron los judíos de Francia que evolucionaron a partir del mismo. En el norte se denominó Zarfático y en el sur Shuadit (también conocido como judeoprovenzal $)^{4}$ y ello, a la luz de lo que veremos más adelante, muy probablemente también guarde parentesco con el judeopiemontés. El perfil de diferenciación idiomática entre el piamontés y el italiano sería el mismo entre el judeopiemontés y el resto de los dialectos que hablaron los hebreos de Italia.

2 Wikipedia; voz "judeolatin".

3 Wikipedia; voz "Lenguas judeo italianas".

4 Wikipedia; Voz "Judeolatín". 
En general, los judíos de Italia no hablaban el idioma hebreo, porque no era corriente entre ellos ${ }^{5}$, a excepción de las necesidades del culto, y desde muy antiguo lo abandonaron para adoptar, según vimos, el latín vulgar, que luego se trasformó en el italiano.

No obstante, la dificultad para encontrar la expresión idiomática adecuada y otras necesidades, como sostiene Diena ${ }^{6}$, hizo que se introdujeran algunas palabras hebreas, que se conjugarían con la gramática italiana, un verdadero cuerpo extraño desde el punto de vista lingüístico, salvo que se sostenga la idea de que los idiomas semitas e indoeuropeo tengan un tronco común ${ }^{7}$. La multiplicidad formada con las respectivas formas dialectales, hizo que estuvieran más influenciadas por el español en el sur, y en el norte por el habla alemana y francesa.

\section{Características del dialecto judeo piamontés}

En relación con el dialecto judeo piamontés, nos hemos permitido incluir, a modo de epígrafe, los textos que encabezan este artículo en italiano, porque rezuman una calidez que sólo ese idioma puede brindar, suavizando lo penoso de una situación histórica que trae, en algunos casos, tristes reminiscencias, a la vez que nos da una mayor comprensión del papel que jugaban los otros dialectos judeo italianos. Estos servían tanto para tener lenguaje en clave en el comercio, como para ocultar significados a la servidumbre $^{8}$ o para vivir en terreno enemigo y comunicarse misteriosamente con los individuos del mismo grupo. El que hablaban en Roma, concretamente, era una respuesta al clima de amenazante opresión en que vivían. El que nos ocupa en este artículo, en cambio, como consecuencia de una situación más favorable, era preferentemente familiar y alusivo, y se utilizaba para conservar las tradiciones ${ }^{9} \mathrm{y}$ mantener cierta alteridad cultural.

5 Scazzochio Sestieri, Lea. Breve Historia del Gheto en Italia. Buenos Aires: Ed. Congreso Judío Latinoamericano, 1971.

6 Diena Paola. Il Giudeo - Piemontese - Tracce Attuali e Testimonianze Sociolinguistichi. Universita Degli Studi di Torino, Tesi di Laurea in Dialettología Italiana.

7 Semerano, Giovanni. (2005) La favola dell'indoeuropeo, Bruno Mondadori Editore.

8 Guttkowski, Helene. Erase una vez en Sefarad. Buenos Aires. Ed. Lumen.

9 Massariello Merzágora Giovanna. Giudeo Italiano: Dialetti Italiani parlati dagli Ebrei. Ed. Pacine. 
El judeo piamontés ("parlate", le llaman los italianos para distinguirlo de las formas dialectales, según veremos) tiene como base el "piemontese", que según especialistas es semejante al occitano o provenzal, el cual, a su vez, origina otros seis dialectos franceses y el catalán, al que -curiosamente- se parecen más que al francés ${ }^{10}$, caracterizado por el consonantismo, con lo cual se aleja decididamente del italiano. Este "piemontese" se hablaba en el Languedoc de Francia y, como muchos hebreos del Piemonte provienen de esa zona, es probable que alguna influencia hayan tenido. Algunos apellidos judeo piemonteses toponímicos, según hemos visto, provienen de asentamientos en esa región como Bedarida (Bédarides Vaucluse), el historiador Momigliano (Montélimar Drôme), Carcassona (Carcasona), o Cavaglione (Cavaglion); otros también evidencian ese origen como los provenzales Vivante; Lattes, Vitta o del Languedoc: Casutto, Foa.

En síntesis, se trata de una estructura menor, de exiguo patrimonio léxico, fundamentalmente oral -pese a la existencia de textos literarios-, sin un sistema fonético, morfológico o sintáctico propio, con pocos lexemas, que se caracteriza por contar con locuciones y expresiones hebreas insertas en el tejido dialectal piamontés. Consecuentemente, el trato fonético y sintáctico era preferentemente piamontés ${ }^{11}$. Esa dimensión disminuida se hace más notoria frente a otros colosos dialectales judíos, como el yiddish, que tuvo amplia expresión literaria, al punto que el premio Nobel de Literatura Isaac Bashevis Singer escribió en esa lengua. Cassuto (1909) destaca muy bien esa diferenciación en cuanto a su importancia si bien hace hincapié en que estas jergas siguen conservando interés glotológico, en forma independiente del número de personas que lo hablen ${ }^{12}$.

9 Ibidem.

10 Enciclopedia Encarta 2000; Microsoft. Voz "Provenzal".

11 Diena; op. cit.

12 Umberto Cassuto afirma: "Infatti, mentre è universalmente nota l'esistenza di un dialetto giudeo-tedesco, quasi nessuno sospetta oltr'alpe che gli ebrei italiani abbiano pure, o almeno abbiano avuto, non dirò un loro dialetto, ma almeno una loro parlata con peculiarie caratteri. Certo, praticamente l'importanza di essa, limitata all'uso quotidiano di poche migliaia di persone, è pressochè nulla di fronte a quella del giudeo-tedesco, il quale è parlato da milioni di individui che bene spesso non conoscono altra lingua, ed ha una propria letteratura, un proprio giornalismo. un proprio teatro, sì da assumere quasi l'importanza di una vera e propria lingua a sè ... è pressochè nulla, se si vuole, anche a paragone di altri dialetti giudaici, del giudeospagnuolo ad esempio, che sono più o meno usati letterariamente; è vero tutto questo, ma dal punto di vista lingüístico tanto vale il giudeo-tedesco, quanto il giudeo-italiano, se così 
A esta falta de autonomía en el plano lingüístico se le agrega que no recogió, aunque sea mínimamente, la cultura local y, aparte de una rara composición en verso de carácter doméstico, que estudió Benvenuto Terracini ${ }^{13}$, nunca traspuso los muros del ghetto y no dejó huellas de importancia. Estas consideraciones nos llevan a concluir -como lo habíamos anticipado- que no tiene la jerarquía de un dialecto, género prejuiciosamente subestimado, porque puede considerarse un idioma. Según Terracini, al constreñirse a ser una lengua de intimidad familiar se reduce su función a la de una jerga, y por tanto es sometido a una progresiva degradación cultural como sucedió efectivamente en este caso, -coincidiendo esta opinión con la de Giuliana Tedeschi (1972) ${ }^{14}$ - porque es el habla de un grupo particular de personas compuesta de términos incomprensibles para otros grupos.

En general, en el judeo piamontés se combinaba el dialecto piamontés, como se dijo, con algunas palabras del idioma hebreo y de otras lenguas que -según vimos- venían del español, del alemán -como Crefoli (kröpfchen, ravioles) y Griben (frituras), que nos traen reminiscencia de la cocina yiddish- y otras de etimología desconocida como Hanichi y Paruscia.

Los estudiosos del habla judeo piamontesa la sistematizaron en glosarios que contienen las frases utilizadas, la estructura, la sintaxis y la fonética del dialecto piamontés vulgar, el cual registra la influencia nítida del dialecto provenzal, que también se adaptaba a esa modalidad idiomática. La influencia del provenzal se daba de una manera doble, por el parentesco que establecimos con el piamontés, y porque es probable que haya sido

vogliamo chiamarlo, giacchè di fronte alla scienza glottologica le varie forme del parlare umano hanno importanza di per sè e non per il numero di persone che le usano o per le forme d'arte in cui vengono adoperate. Piuttosto, una notevole differenza fra il giudeo-tedesco e il giudeo-italiano, che ha valore anche per il riguardo scientifico, è che, mentre quello è tanto diverso dalla lingua tedesca da costituire un dialetto a sè stante, questo invece non è essenzialmente una cosa diversa dalla lingua d'Italia, o dai singoli dialetti delle varie provincie d'Italia (...) era naturale che il gergo giudeo-italiano in breve volger di tempo sparisse... ("Parlata ebraica" Vessillo Israelitico 57, 1909, 255-256)

13 Terracini Benvenuto Il Problema Della Traduzione By Benvenuto Terracini. Pdf ePub.Uploaded on 01/15/2017

${ }^{14}$ Tedeschi, G. Lingua grammatical stile. G.B. Petrini, 1972 
prestatario de la forma de hablar de los hebreos, en su paso por esa región francesa, procedentes de España. Es el caso del vocablo mencionado, "creada "(doméstica), que según algunos autores, si bien ha tenido origen en el español, registró un paso por ese dialecto, (creat), con un significado algo distinto.

Algunos glosarios ${ }^{15}$ hacían una subclasificación en función del grado de deformación de la palabra original, por lo cual me ocuparé de las que se pronuncian según el trato fonético piamontés. Hay que señalar que esos vocablos de origen hebreo se conjugaban a partir de la raíz hebrea siguiendo la desinencia de la norma local, es decir, se "piemontesaban". Tenemos, por ejemplo, la palabra "chaborie" (comer) que conjugada resulta: Chaborioma, il chaborie, s`chaborieissa. Además, y con frecuencia, la formación del femenino desde el masculino, tanto para los sustantivos como para los adjetivos, resultaba de la aplicación de sufijos propios del piamontés.

Veamos a modo de ejemplo algunos vocablos hebreos integrando distintas oraciones del dialecto tratado con sus respectivas traducciones (gentileza de la profesora Ana María Filippa):

AGADÁ (narración): “...che bel güst di la gadà...” / "Qué placer contar una historia”. BERAHÁ (bendición): “T l as nen di braha..." / "No diste la bendición".

DAVAR (silencio o no hablar; uno de los términos más utilizados); "Dabré davar..." "Deberé callar"; "Dabra davar ch`a j’è $i$ meghelim" (espía). "Hay que callar que hay espías"; "Custa mnestra a sa `d davar OLAM" (mundo; ejemplos con esa palabra: "A va mal stu ulam..." / "Va mal este mundo", “...robe dell alter olam..." / "Cosas del otro mundo"; "Davar seba`olam cha la banà à chamna...".

ANIÚD (miseria) "T è d'ausogn a travaié a ura d'sabat: a'tciami al aniüd" / "Tienes necesidad de trabajar el sábado: llamas a la miseria".

AREL (crédulo); "Valu a cunté ai arelim" / "Andá a contarle a los crédulos" (forma plural que se forma con el sufijo "im").

AVÓN (pecado); "S'a l'avia di avunod lá spürgaie túti" / "Si tenía pecados, los purgó a todos".

15 Ibid. 
MANOD(dinero); "metá cônseil e metá madod" / "Mitad consejos y mitad dinero"; “...e par urvi presto i sarot gnor Luzatti l'sborzá i manot...”

MAZA O MASSA (masa ácima); “Le massot valut pu nenta, i biscott smíód polenta..." /

"Las masan ya no valen nada, los biscochos parecen de polenta".

RUBI' (rabino; doctor); "Nun a's fa pu nenta 'd bon dop ch a j'è robè Ghiron..." / "Nadie hace ya nada bien, desde que está el rabino Ghiron"; "Sél Rubì a d na dà mia sur Rubi s'na vada via...".

SCHÜRCHNA (mueca); “...l'ambissium la pu scurnada al a fane sbagliè strada...” / "La ambición con una mueca, nos hizo errar el camino".

HAZÀN: (cantor oficiante); "Bütlu in testa al Hazan..." / "Decíselo al cantor".

SICÒR (borracho); “...chiel lì a l'è n sicor..." / "Ese es un borracho" (al parecer existe la opinión que el vocablo hebreo piemontés sicurie está cruzado con el provenzal chicolhá).

SUÁ (excremento); "A l'éna suá muntà an scagn..." / "Es un excremento subido a un escaño" A l'éna suá...".

TOV (bueno); "Nun l`e'il frac negher e 'l culet nsu ca fan 'l bel adam, ma i maásim tovim e la virtù..." / "No es el frac negro y el cuello levantado lo que hacen a un hombre bello, sino la virtud".

TU ÉVA (iglesia católica); "Stu iüdi l'è andait an tu 'eva..." / "Este judío fue a una iglesia".

\section{Trazos actuales y testimonios sociolingüísticos}

Según Casutto (1909), la desaparición de la jerga judeo italiana en general es inevitable; en similar sentido, Primo Levi, refiriéndose al judeo piamontés, afirma que su interés histórico es exiguo porque nunca fue hablado por más de un par de miles de personas, pero destaca su importancia en relación con lo humano, como ocurre con todos los lenguajes de confines y transiciones.

Diena trató de determinar sus últimas huellas, que por los años 80 eran muy tenues, y entiende que por su escasísima vitalidad hubiera resultado muy ardua su recuperación, hasta tal punto que para confeccionar un glosario tuvieron que hacer notables esfuerzos mnemotécnicos. Por otra parte, el investigador tuvo también importantes dificultades 
etimológicas por la continua deformación de las palabras propia de ese proceso de decadencia. Del universo de la población hebrea torinense, la muestra hecha en relación con la gente de más de sesenta años, solamente se encontraron setenta personas que poseían suficiente memoria histórica y lingüística. Al tratar de encontrar las causas de la profunda decadencia, considera que al producirse la cancelación del ghetto la mayoría de los núcleos hebraicos entraron en tensión con la intención de integrarse totalmente a la sociedad circundante y esos lenguajes particulares constituían un obstáculo para la fluidez de las relaciones sociales y culturales que querían entablar. Por esa razón se trasformó en ocasional y se produjeron deslizamientos semánticos en relación con el significado original de las palabras hebreas, lo cual, unido a las deformaciones que hemos visto, le quitaban vigencia.

Además, a la opinión Diena, acerca de la asimilación, agregaríamos que si esta es una causa de la decadencia, la que correspondió a Italia no fue una adaptación más de los hebreos a la modernidad, como ocurrió en todos los países, sino un proceso integral y vertiginoso. Hacer un dimensionamiento de ese proceso en Italia escapa a este trabajo. Solamente diremos que la situación del antisemitismo en Italia fue tan singular que hizo que ese patrón de conducta que importó el antisemitismo moderno tuviera en esa nación una excepción, con lo cual la causa mencionada de la decadencia identitaria, en este caso relacionada con un lenguaje, en Italia tiene que haber sido más intensa que en otras geografías.

Por último, debe considerarse también la regresión de los dialectos en general, que se ven desplazados por los idiomas oficiales en su inevitable tarea de nivelación. No obstante, si algunos entienden, con buenas razones, que este dialecto es un arcaísmo histórico, hay que destacar que a mediados de siglo XX aún conservaba alguna vigencia, como lo demuestra una publicación reciente de Cavaglion (1998) ${ }^{16}$ que narra las peripecias de un medico judío perteneciente a la "buona" burguesía torinesa durante la segunda guerra mundial. De la misma surge que, en 1944, pese a estar casado con una católica practicante, en casa del médico se hablaba dialecto, utilizando expresiones como: “Ancöi am sentu taréf (hoy no me siento bien)" y la palabra hebrea "taréf', que

16 Cavaglion Alberto. Per Vía Invisibile. Bologna, Ed. Il Mulino, 1998. 
significa devorado o despedazado. Por ello estimamos que, tratándose de una época no tan lejana, y proviniendo de un hogar compuesto por un matrimonio mixto de la alta clase media, esa manera de hablar no había caído totalmente en desuso aún, aunque no tardaría en llegar a ese estado.

\section{Préstamos lingüísticos}

Hay otro aspecto muy interesante que está emparentado con la modalidad lingüística que tratamos: el intercambio y los préstamos desde la lengua hebraica al dialecto de los piamonteses cristianos, signo de una larga permanencia de relaciones amistosas entre los dos grupos a pesar de los ghettos y de la discriminación ${ }^{17}$. Según comenta el profesor de la Universidad de California y editor del Boletín Piemontesi nel mondo of Northern California Andrew Canepa, el lenguaje del ghetto ha sido, en alguna medida, tributario del piamontés vulgar; es decir, algunas palabras piamontesas tienen raíces hebreas.

Así es como existen algunos vocablos incorporados al dialecto piamontés en su variante vercellesa ${ }^{18}$ que provienen del hebreo, como taréf (ya vimos su significado), magon (tristeza), cacam (persona con alto cargo, y también soberbia). En el dialecto casalese también ingresaron palabras hebreas como nainé (guardar), y banim (muchachos o hijos) $)^{19}$.

17 Sarasso, Terenzio. Storia degli Ebrei a Vercelli. Ed. Comunitá Israelitica di Vercelli; (Vercelli, 1974). "É questo il periodo in cui la colonia giudaica e vercellese si integra e si amalgama sempre maggiormente con la cittadinanza a tal punto che tra l'una e l'altra si verifica un fenomeno linguistico di reciproca assimilizione, un continuo intercambio di termine lessicali e di particolari strrutture morfologiche...".

18 Ibidem.

19 Segre Augusto; Memorie di Vita Ebraica-Casale Monferrato - Roma - Gerusalemme. Roma, Ed. Bonacci, 1979. 


\section{Bibliografía}

Cassuto Umberto "Parlata ebraica." Vessillo Israelitico 57 (1909):

Colorni Vittore; “Cognomi Ebraici Italiani a Base Toponomastica Straniera" en Italia Judaica; Roma, 1989.

Cavaglion Alberto. Per Vía Invisibile. Bologna, Ed. Il Mulino, 1998

Diena Paola. "Ebrei a Torino". Richerche per il Centenario Della Sinagoga, 1984.

Diena Paola. Il Giudeo Piemontese. Trace Attuali e Testimonianza Sociolinguistiche. Ed. Allemandi, 1984.

Guttkowski Helene. Érase una vez en Sefarad. Buenos Aires, Ed. Lumen.1999.

Massariello Merzágora Giovanna. Giudeo Italiano: Dialetti Italiani parlati dagli Ebrei. Ed. Pacine, 1977.

Scazzochio Sestieri, Lea. Breve Historia del Gheto en Italia. Buenos Aires, Ed. Congreso Judío Latinoamericano, 1971.

Segre Augusto. Memorie di Vita Ebraica-Casale Monferrato - Roma-Gerusalemme. Roma, Ed. Bonacci, 1979.

Virgilio, Alberto. Como si parla a Torino. Torino, Lattes, 1897. 